\title{
Exit Strategies in Natalizumab-Treated RRMS at High Risk of Progressive Multifocal Leukoencephalopathy: a Multicentre Comparison Study
}

\author{
Aurora Zanghì ${ }^{1} @$ - Antonio Gallo ${ }^{2} \cdot$ Carlo Avolio $^{3} \cdot$ Rocco Capuano $^{2} \cdot$ Matteo Lucchini $^{4} \cdot$ Maria Petracca $^{7}$. \\ Simona Bonavita ${ }^{6} \cdot$ Roberta Lanzillo $^{7}$. Diana Ferraro ${ }^{8}$. Erica Curti $^{9} \cdot$ Maria Buccafusca $^{10}$. Graziella Callari $^{11}$. \\ Stefania Barone ${ }^{12}$. Giuseppe Pontillo ${ }^{13,14}$. Gianmarco Abbadessa ${ }^{15}$. Valeria Di Francescantonio ${ }^{3}$. \\ Elisabetta Signoriello ${ }^{16}$. Giacomo Lus $^{16} \cdot$ Patrizia Sola $^{8} \cdot$ Franco Granella $^{17,9} \cdot$ Paola Valentino $^{12}$. \\ Massimiliano Mirabella ${ }^{4,5} \cdot$ Francesco Patti $^{1} \cdot$ Emanuele D'Amico $^{1}$ (D)
}

Accepted: 9 March 2021 / Published online: 12 April 2021

(c) The Author(s) 2021

\begin{abstract}
The main aim of the study is to evaluate the efficacy and safety profile of ocrelizumab (OCR), rituximab (RTX), and cladribine (CLA), employed as natalizumab (NTZ) exit strategies in relapsing-remitting multiple sclerosis (RRMS) patients at high-risk for progressive multifocal leukoencephalopathy (PML). This is a multicentre, retrospective, real-world study on consecutive RRMS patients from eleven tertiary Italian MS centres, who switched from NTZ to OCR, RTX, and CLA from January 1st, 2019, to December 31st, 2019. The primary study outcomes were the annualized relapse rate (ARR) and magnetic resonance imaging (MRI) outcome. Treatment effects were estimated by the inverse probability treatment weighting (IPTW), based on propensity-score (PS) approach. Additional endpoint included confirmed disability progression (CDP) as measured by Expanded Disability Status Scale and adverse events (AEs). Patients satisfying predefined inclusion and exclusion criteria were 120; 64 switched to OCR, 36 to RTX, and 20 to CLA. Patients from the 3 groups did not show differences for baseline characteristics, also after post hoc analysis. The IPTW PS-adjusted models revealed that patients on OCR had a lower risk for ARR than patients on CLA $\left(\operatorname{ExpB}_{\mathrm{OCR}} 0.485\right.$, CI 95\% 0.264-0.893, $\left.p=0.020\right)$. This result was confirmed also for 12-month MRI activity ( $\left.\operatorname{ExpB}_{\mathrm{OCR}} 0.248 \mathrm{CI} 95 \% 0.065-0.948, p=0.042\right)$. No differences were found in other pairwise comparisons (OCR vs RTX and RTX vs CLA) for the investigated outcomes. AEs were similar among the 3 groups. Anti-CD20 drugs were revealed to be effective and safe options as NTZ exit strategies. All investigated DMTs showed a good safety profile.
\end{abstract}

Key Words Natalizumab $\cdot$ Ocrelizumab $\cdot$ Rituximab $\cdot$ Cladribine $\cdot$ Exit strategy $\cdot$ Disease activity

\section{Introduction}

Natalizumab (NTZ) has improved the possibilities to treat highly active relapsing-remitting forms of multiple sclerosis (RRMS) patients (1).

However, a long exposure to NTZ treatment in anti-JC virus (JCV)-seropositive patients expose them to a higher risk to develop progressive multifocal leukoencephalopathy (PML), a serious and potentially lethal opportunistic brain

Emanuele D’Amico

emanuele.damico@unict.it

Extended author information available on the last page of the article infection [2-5]. To manage PML risk, a magnetic resonance imaging (MRI) monitoring every $3-4$ months has been recommended for JCV-seropositive patients on NTZ treatment for more than 18 months [5]. Furthermore, although several retrospective studies have investigated the effect of extended interval dose (EID) on reducing PML risk, the reliability of their conclusions is limited by the nonrandomized designs, and the extreme variable definitions of EID (ranging from 5 to 8 weeks) [5].

All above considered, a therapeutic switch in patients who respond to NTZ but are exposed to a high PML risk represents an important and increasingly frequent therapeutic challenge in MS clinical practice. Since highly effective drugs have been licensed for the treatment of highly active RRMS, there is 
an urgent need of clinical-MRI data to develop guidelines addressing/regarding exit strategies to follow in JCV-positive NTZ-treated RRMS patients at high risk of PML [6-8].

The decision to switch to another drug is always shared between neurologist and patient, and it should derive from the balance of several factors, including the risk of side effects, the maintenance of a good clinical-MRI response, and the occurrence of a clinical and radiological rebound that has been frequently observed early after NTZ discontinuation [3, 9-17].

About therapeutic options after NTZ withdrawal, the drugs targeting CD20 + B cells (rituximab (RTX) and ocrelizumab (OCR)) have proven to be very effective in suppressing inflammatory activity in RRMS [18], although not associated with a significant PML risk [19].

More recently, cladribine (CLA) tablets have been approved for highly active RRMS, but scarce data are available on the use of CLA after NTZ [7, 20].

On this background, the aim of the present multicentre real-world study was to compare the effectiveness, tolerability, and safety of a therapeutic switch to OCR, RTX, or CLA in RRMS patients treated with NTZ who were considered responder to such treatment that needed or required to stop NTZ for the high risk of PML.

\section{Methods}

\section{Setting and Participants}

In this retrospective observational study, we collected prospective clinical and MRI data from RRMS patients followed at 11 tertiary Italian MS centres. In detail, we identified adult RRMS patients who switched from NTZ to OCR, RTX, and CLA from January 1st, 2019, to December 31st, 2019.

Inclusion criteria were: 1 ) $\geq 18$ years of age, 2) a diagnosis of RRMS according to McDonald revised diagnostic criteria [21]; 3) no evidence of clinical and radiological activity in the last 12 months of NTZ treatment (with a standard or EID regimen); and 4) washout period from NTZ treatment not longer than 12 weeks.

We considered for the analysis only RRMS patients who switched form NTZ for the following safety reasons:

1. a JCV index $\geq 1.5$ and more than $24 \mathrm{NTZ}$ infusions

2. patient's decision to discontinue NTZ for the fear of an increased risk of PML, even if the positive JCV index was $<1.5$.

The decision to stop NTZ was reached after a consultation between the neurologist and the patient, during which the risks and benefits of continuing or interrupting NTZ were clearly explained and discussed.

\section{Procedures and Outcomes}

Patients were treated in accordance with treatment procedures and guidelines approved by European and Italian Medicines Agencies.

In detail, OCR is administered at the dosage of $600 \mathrm{mg} /$ intravenous, and the first 2 infusions-each of $300 \mathrm{mg}-$ are given 2 weeks apart and subsequent $600-\mathrm{mg}$ infusions are given every 6 months [22].

RTX is used as off-label treatment in highly active RRMS patients [23]. It is administered as an intravenous infusion in doses of $1000 \mathrm{mg}$ [24]. Subsequent doses and timing of administration are usually every $6-12$ months, but no consensus guidelines exist. Among our RRMS patients, the interval between the first and second infusions was on average 7 months (range 6-9).

CLA tablets are administered in 2 treatment courses $\sim 1$ year apart [7]. The recommended cumulative dosage is $3.5 \mathrm{mg} / \mathrm{kg}$ body weight administered orally and divided into 2 yearly treatment courses $(1.75 \mathrm{mg} / \mathrm{kg}$ per treatment course). Each treatment course is divided into 2 treatment cycles [7]. The first treatment course (year 1) is structured as follows: a first cycle (month 1 ) that starts at any given time and a second cycle (month 2) which starts 23-27 days after the last dose ( 1 month after beginning first cycle).

Data were recorded retrospectively (including data until 12 months before NTZ starting, time on NTZ, and the washout period) and prospectively (until the last available visit of follow-up) from the beginning of 1 the 3 investigated drugs (the index date).

The data entry portal was iMed ${ }^{\circledR}$ software's (iMed, Merck Serono SA - Geneva, Switzerland). Data were extracted on September 30th, 2020.

Disability was assessed by Expanded Disability Status Scale (EDSS) by a neurostatus-certified MS specialist. MRI data were acquired on 1.5-T scanners (the same at each centre from baseline to the end of the follow-up) and included T2and pre- and postcontrast T1-weighted sequences [25]. Postcontrast $\mathrm{T} 1$-weighted sequences were acquired after intravenous injection of gadolinium contrast agent $(0.1 \mathrm{mmol} / \mathrm{kg})$. A cerebral MRI acquired within 30 days before the treatment start (during the washout period) was considered the baseline MRI, and the number of brain T2-, pre-, and postcontrast T1 lesions was recorded. Follow-up MRIs to assess disease activity were acquired at 6 and 12 months after the start of post-NTZ treatment. 


\section{Study Endpoints}

The primary study outcome was the annualized relapse rate (ARR) on investigated drugs. Additional endpoints included MRI activity after 12 months and confirmed disability progression (CDP) as measured by EDSS until the last follow-up.

Safety profile of the investigated DMTs was also investigated and reported.

A relapse was defined as the development of new symptoms or exacerbation of existing symptoms that persisted for $\geq 24 \mathrm{~h}$, in the absence of concurrent illness or fever, and occurred $\geq 30$ days after a previous relapse. ARR was defined as total number of relapses divided by patient-months on therapy.

CDP was defined as an increase in EDSS by $\geq 1.5$ points for those with a baseline EDSS score of 0 , by 1 or more points for a baseline score of $\leq 5.5$, or by 0.5 points for a baseline score of $>5.5$, which was sustained for 12 weeks or longer. EDSS recorded within 30 days after the onset of a relapse were excluded.

MRI activity was considered new T1-gadolinium enhancing brain lesion and/or a new or newly enlarging T2 brain lesion [26].

We defined EID if the mean interval between doses were $\geqslant 5$ weeks and standard interval dose (SID) if the mean interval between doses were $<5$ weeks. The 5 -week cut-off was defined a priori being the midpoint between SID (4 weeks) and EID (6-8 weeks) [27]. All patients received SID NTZ for at least 1 year, and after that, some were switched to an EID regimen due to the risk of PML.

We collected data on the safety and tolerability, reporting the frequency of Adverse Events (AEs) in accordance to EMA definition [28]. Registered AEs were severe infections requiring medication, except for uncomplicated lower urinary tract infections; AEs causing discontinuation of therapy; and AEs related to each infusion of OCR/RTX or the first dosing of CLA cycle (both reported separately).

\section{Protocol Approval Standard, Registrations, and Patient Consents}

The study protocol was approved by the local ethics committee (Comitato Etico Catania 1 no. 140/2020/PO) of the coordinating centre (Policlinico Vittorio Emanuele, Catania, Italy), and patients provided written informed consent. The study was conducted in accordance with the ethical principles of the Declaration of Helsinki and with the appropriate national regulations.

\section{Statistical Analysis}

All patient characteristics summary statistics are reported in terms of frequencies (\%) for categorical variables, mean standard deviation (S.D.), or median with interquartile range (IQR) for continuous variables. The Kolmogorov test was used to verify data distribution. According to this latter, parametric or nonparametric test was employed. The Bonferroni test was used for post hoc analysis.

According to the Akaike information criterion, we selected the model with the best statistical inferential properties. All the models were estimated using the Breslow's tie correction.

To consider the imbalance of the 2 groups, a propensity score (PS) was calculated as the following.

A logistic regression was performed to score all patients according to the treatment $(\mathrm{OCR}=1 \mathrm{vs} \mathrm{CLA}=0, \mathrm{OCR}=1$ vs $\mathrm{RTX}=0$ and $\mathrm{RTX}=1$ vs CLA $=0$ ) used as independent variable and the following covariates at baseline: age, sex, EDSS in the year prior to switch to new DMT, number of NTZ infusions, and EID during the NTZ treatment as covariates.

Inverse probability of treatment weight (IPTW) and the stabilized inverse probability of treatment weight (SIPTW) were also calculated. HRs and CI 95\% were calculated.

Two generalized regression models IPTW PS-adjusted were performed to evaluate relationship between: I) ARR and treatment groups and II) MRI activity after 12 months and treatment groups. The generalized equation models employed were adapted according to the nature of variables, respectively, linear (for ARR expressed as a continuous variable) and logistic binary (for MRI activities, expressed as dichotomic).

CDP, as measured by EDSS, was compared using a contingency table.

SPSS version 21.0 was used for all analyses (IBM SPSS Statistics 21, IBM $\odot$, Armonk, NY, USA).

\section{Results}

From a total cohort of 980 RRMS patients treated with NTZ in the enrolled centres, 170 stopped NTZ for any reasons during the index window, and 120 fulfilled the required criteria. Out of them, 64 switched to OCR, 36 switched to RTX, and 20 switched to CLA (Fig. 1). Demographical and clinical characteristics of the whole cohort and groups are reported in Table 1. Overall, the entire 


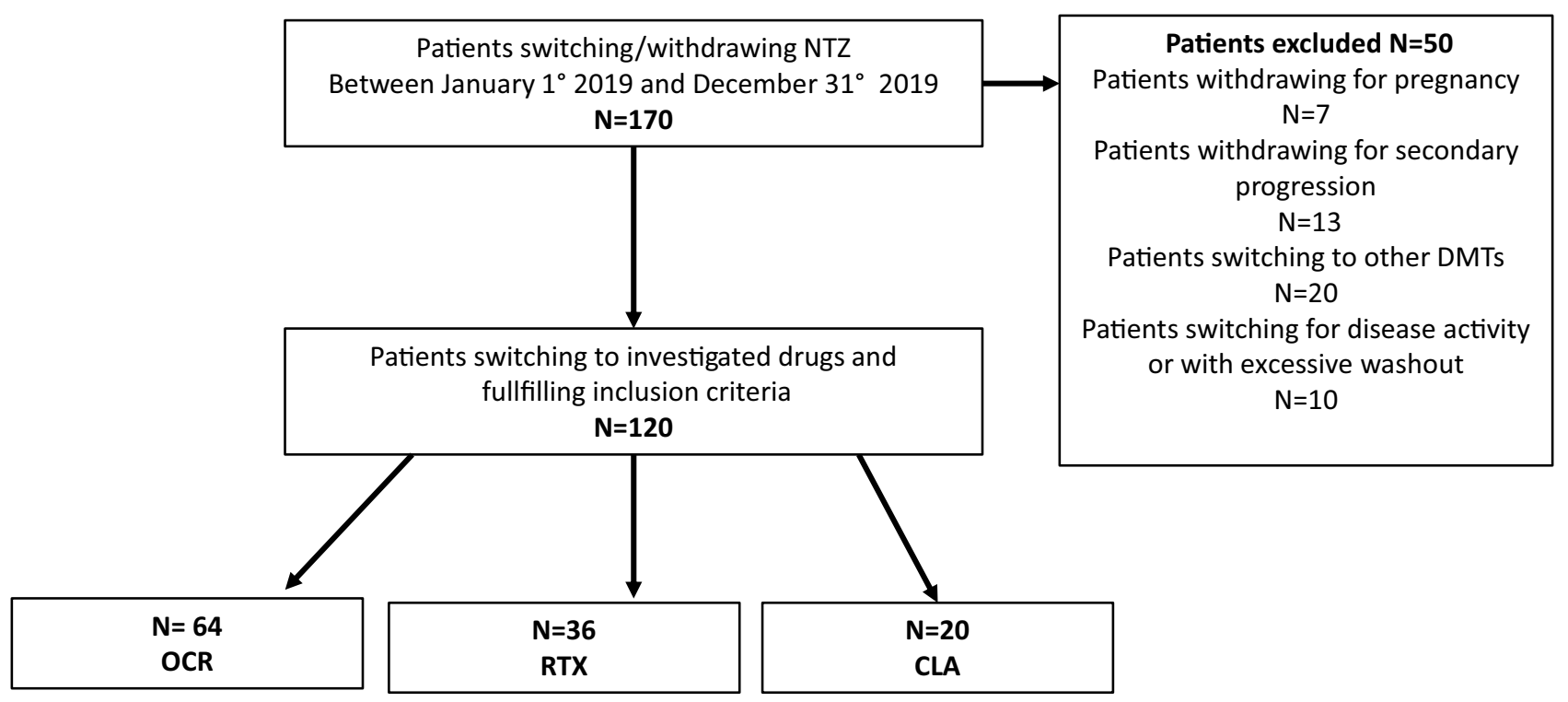

Fig. 1 Patients' selection flow chart. CLA = cladribine; NTZ = natalizumab; OCR = ocrelizumab; RTX = rituximab

cohort did not show differences for baseline characteristics (Table 1). After post hoc test, the results were confirmed.

Table 2 shows the main clinical and radiological findings after switch among the 3 groups.

The estimated means for ARR showed a trend of significativity among the 3 groups, with value of 0.001 for patients on OCR, 0.308 for patients on RTX, and 0.500 for patients on CLA $(p=0.053)$.

The generalized regression model IPTW PS-adjusted revealed that patients on OCR had a lower risk for ARR than patients on CLA $\left(\operatorname{ExpB}_{\mathrm{OCR}} 0.485\right.$ CI 95\% 0.264-0.893, $p=0.020)$.

No differences were found for the investigated out-

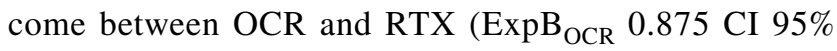

$0.749-1.021, p=0.089)$ and between RTX and CLA $\left(\mathrm{ExpB}_{\mathrm{RTX}} 0.858\right.$ CI 95\% 0.640-1.149 $\left.p=0.304\right)$ (Fig. 2).

The generalized regression model IPTW PS-adjusted revealed that patients on OCR had a lower risk for MRI activity than patients on CLA $\left(\operatorname{ExpB}_{\mathrm{OCR}} 0.248\right.$ CI $95 \%$ 0.065-0.948, $p=0.042$ ).

No differences were found for the investigated outcome between OCR and RTX (ExpB ${ }_{\text {OCR }} 1.247$ CI 95\% 0.573-2.717, $p=0.578$ ) and between RTX and CLA $\left(\mathrm{ExpB}_{\mathrm{RTX}} 1.240 \mathrm{CI} 95 \% 0.263-5.851 p=0.786\right)$ (Fig. 2).

The CDP at the last follow-up did not differ among the 3 groups $(p=0.953)$.

No patient received a diagnosis of PML. Fourteen patients reported AEs within the first 12 months of treat-

Table 1 Baseline characteristic among the 3 groups

\begin{tabular}{|c|c|c|c|c|}
\hline Variables $^{\circ}$ & OCR (64) & RTX (36) & CLA (20) & $p^{*}$ \\
\hline Female $n,(\%)$ & $42,(65.6)$ & $26,(72.2)$ & $13,(65)$ & ns \\
\hline Age at disease onset ( \pm mean, S.D.) (year) & $24.4 \pm 9.5$ & $23.7 \pm 9.9$ & $26.5 \pm 10.2$ & ns \\
\hline Number of DMTs before NTZ & $1.3 \pm 1.1$ & $1.7 \pm 1.1$ & $1.1 \pm 0.8$ & ns \\
\hline Number of relapses $12 \mathrm{~m}$ before NTZ start & $1.6 \pm 1.1$ & $1.4 \pm 1.2$ & $1.6 \pm 0.9$ & ns \\
\hline Number of T2-weighted brain lesions $12 \mathrm{~m}$ before NTZ start & $28.2 \pm 16.1$ & $23.8 \pm 12.5$ & $21.6 \pm 13.1$ & ns \\
\hline Number of T1-Gd + brain lesions $12 \mathrm{~m}$ before NTZ start & $1.6 \pm 2.2$ & $1.5 \pm 1.7$ & $1.0 \pm 1.2$ & ns \\
\hline EDSS at NTZ start, median (interquartile range) & $3.0(2.0-4.5)$ & $2.5(2.0-4.0)$ & $2.0(1.0-3.0)$ & ns \\
\hline Number of NTZ infusions & $35.1 \pm 26.9$ & $40.3 \pm 24.9$ & $26.7 \pm 15.8$ & ns \\
\hline $\operatorname{EID}(n, \%)$ & $25(39.1)$ & $11(30.6)$ & $6(30)$ & ns \\
\hline EID duration (weeks) & $16 \pm 3.2$ & $15 \pm 4.7$ & $14 \pm 5.1$ & ns \\
\hline Washout period (weeks) & $8 \pm 4.2$ & $7 \pm 3.9$ & $6 \pm 2.9$ & ns \\
\hline
\end{tabular}

Data are expressed as mean \pm S.D. when otherwise specified

$D M T$ disease modifying therapy; EDSS Expanded Disability Status Scale; EID extended interval dose; NTZ natalizumab

*via $\chi 2$, Fisher exact test or ANOVA according to the nature of variables 
Table 2 Clinical and radiological findings among the 3 groups after switch

\begin{tabular}{|c|c|c|c|c|}
\hline Variables $^{\circ}$ & OCR (64) & RTX (36) & CLA (20) & $p^{*}$ \\
\hline Patients relapsing during treatment $n(\%)$ & $5(7.8)$ & $5(13.9)$ & $4(20)$ & ns \\
\hline Patients with more than 1 relapse during treatment $n(\%)$ & 0 & $3(8.3)$ & $3(15)$ & 0.017 \\
\hline Median time to first relapse median ( $\mathrm{q} 1-\mathrm{q} 3$ ) (months) & $3(2-3)$ & $6(3-8)$ & $3(2.7-3.7)$ & ns \\
\hline EDSS at baseline median (q1-q3) & $3.0(2.0-4.5)$ & $4.0(2.0-4.5)$ & $2.0(1.0-3.0)$ & ns \\
\hline EDSS after 6 months median (q1-q3) & $3.0(2.0-4.5)$ & $4.0(2.0-4.5)$ & $2.0(1.0-4.0)$ & ns \\
\hline EDSS after 12 months median (q1-q3) & $3.0(2.0-4.5)$ & $3.5(1.5-4.0)$ & $3.0(1.5-5.0)$ & ns \\
\hline EDSS after 18 months median (q1-q3) & $3.0(2.0-4.5)$ & $3.5(1.5-4.0)$ & $3.0(1.5-5.0)$ & ns \\
\hline Patients with CDP at last follow-up $n(\%)$ & $5(7.8)$ & $3(8.3)$ & $2(10)$ & ns \\
\hline $\begin{array}{l}\text { Patients with increased lesions load on T2-weighted or T1 } \\
\text { Gad + weighted brain MRI lesions after } 6 \text { months } n(\%)\end{array}$ & $5(7.8)$ & $3(8.3)$ & $4(20)$ & ns \\
\hline $\begin{array}{l}\text { Patients with increased lesions load on T2-weighted or T1 } \\
\text { Gad + weighted brain MRI lesions after } 12 \text { months } n(\%)^{* *}\end{array}$ & $6(9.3)$ & $6(16.7)$ & $4(20)$ & ns \\
\hline Follow-up in months median (q1-q3) & $18(15-19)$ & $17(14-20)$ & $16(13-18)$ & ns \\
\hline
\end{tabular}

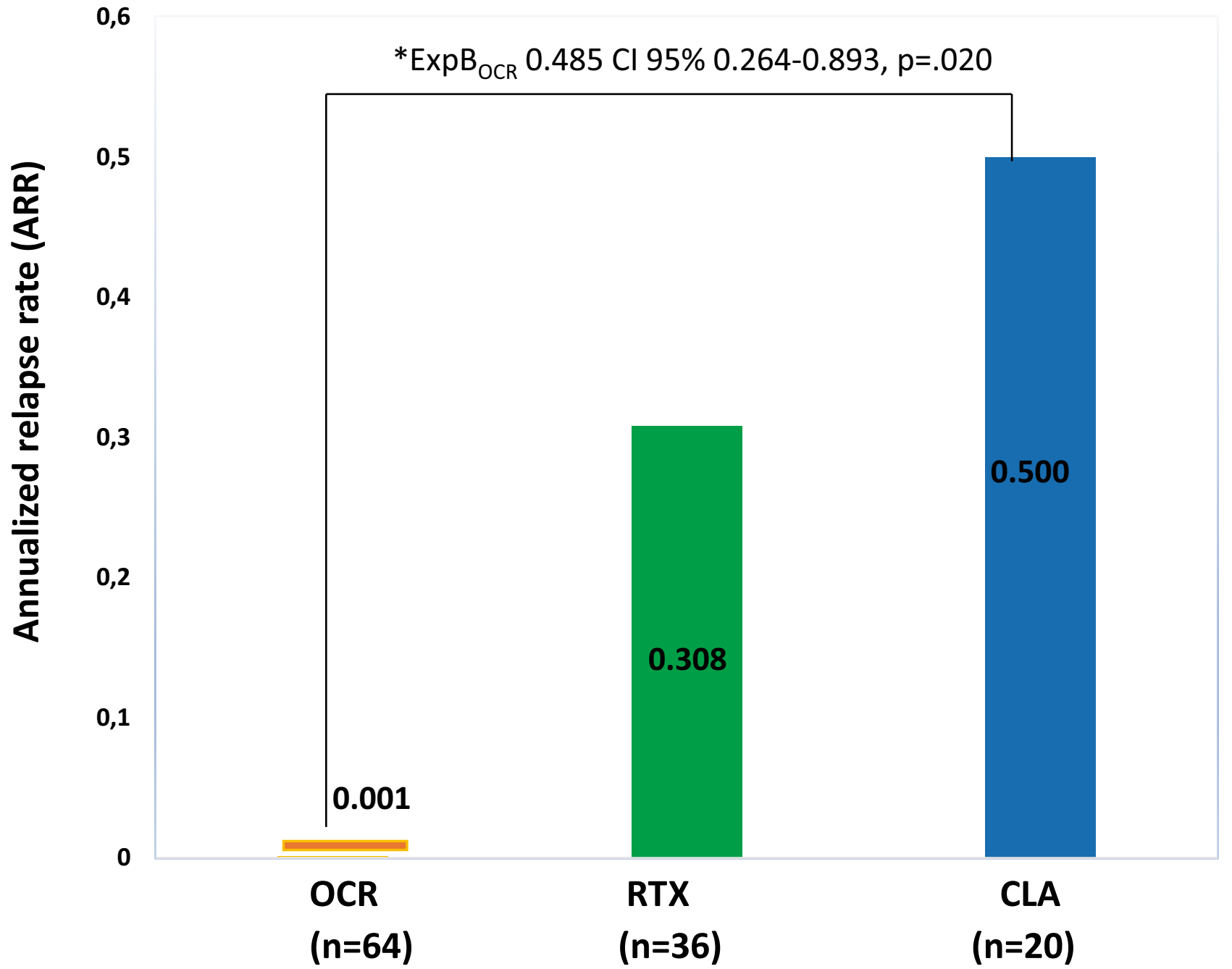

Fig. 2 ARR endpoint (asterisk). The treatment effects were explored by a propensity-score adjustment in quintiles for age, sex, and EDSS in the year prior to switch to new DMT, number of NTZ infusions, and EID during the NTZ treatment. ARR = annualized relapse rate; $\mathrm{CI}=$ confidence interval; $\mathrm{CLA}=$ cladribine; $\mathrm{NTZ}=$ natalizumab; $\mathrm{OCR}=$ ocrelizumab; RTX $=$ rituximab 
Table 3 Adverse events among the 3 groups

\begin{tabular}{|c|c|c|c|}
\hline & $\begin{array}{l}\text { OCR } \\
(n=64)\end{array}$ & $\begin{array}{l}\text { RTX } \\
(n=36)\end{array}$ & $\begin{array}{l}\text { CLA } \\
(n=20)\end{array}$ \\
\hline \multicolumn{4}{|l|}{ AE, within 12 months } \\
\hline Patients with AEs & $\begin{array}{l}3 \text { ( } 2 \text { urinary infec- } \\
\text { tions, } \\
1 \text { gastrointestinal } \\
\text { infection) }\end{array}$ & 1 (urinary infection) & $\begin{array}{l}1 \text { (1 genitourinary } \\
\text { infection) }\end{array}$ \\
\hline \multicolumn{4}{|l|}{ First-dosing AEs } \\
\hline Patients with first-dosing AEs & $\begin{array}{l}5 \text { (headache, flushing, } \\
\text { articular pain) }\end{array}$ & $\begin{array}{l}3 \text { (headache, flushing, } \\
\text { articular pain) }\end{array}$ & $\begin{array}{l}1 \text { (seborrheic dermati- } \\
\text { tis of the scalp) }\end{array}$ \\
\hline \multicolumn{4}{|c|}{ AEs resulting in DMT discontinuation, within 12 months } \\
\hline Patients discontinuing for safety concerns & 0 & 0 & 0 \\
\hline
\end{tabular}

AEs adverse events; $O C R$ ocrelizumab; $R T X$ rituximab; $C L A$ cladribine; $D M T$ disease modifying therapy ment. Out of them, severe infections were reported in 3 patients on OCR, 1 on RTX, and 1 on CLA (Table 3).

First-dosing AEs were reported in 5 on OCR and in 3 patients on RTX. One patient on CLA after the first cycle reported seborrheic dermatitis of the scalp. None of the AEs reported lead to DMT discontinuation.

\section{Discussion}

Our study revealed a lower risk of experiencing relapses and new MRI activity for patients that switched from NTZ to OCR than CLA. Contrariwise, no differences were found between those switching to RTX and CLA.

Regarding CDP, no differences were found, at the end of the follow-up, between the 3-switching group.

Overall, all DMTs revealed a good safety profile with no cases of PML.

The increased risk of PML in NTZ long-treated patients who show JCV antibodies positivity represents a matter of great concern in clinical practice. Different schemes of NTZ monitoring and/or administration, as EID regimen, have been proposed and evaluated, but they do not cancel PML risk and its consequences [5, 29, 30].

We could speculate that our results reflect the different mechanisms of action and pharmacodynamics of the investigated DMTs.

Little is known about the reasons of clinical and radiological rebound after NTZ discontinuation. It was considered the role of increased percentage of activated $T$ cells producing cytokines in the peripheral circulation during NTZ treatment [31].

Real-world observational data about OCR as NTZ exit strategy are recent, whilst efficacy and safety of RTX have been highlighted since 2016 [9, 32-35].

A recent study investigated 42 RRMS patients who switched to OCR from NTZ after EID (5-8 weeks) and who were followed up for 6 months, clinical relapses occurred during the first 3 months of observation in 5 patients, and the EDSS remained stable in $38(90 \%)$ patients. No serious AEs were described [34].

The most relevant observational study compared patients switching from NTZ to RTX ( $n=114)$ to patients switching to fingolimod $(n=142)$ with an average follow-up of 1.5 years. Here, relapses occurred in $1.8 \%$ of RTX-treated patients compared with $17.6 \%$ of those who switched to fingolimod. The rates of AEs (5.3\% vs $21.1 \%$ ) and treatment discontinuation ( $1.8 \%$ vs $28.2 \%$ ) were also lower in RTX groups. These results have been confirmed by a number of recent case series [19, 32, 33].

In our cohort, OCR was associated to lower relapse and lower MRI activity than CLA.

The comparative efficacy of CLA versus other DMTs in naïve patients has been analysed through meta-regression and matching-adjusted indirect treatment comparison approaches [36]. In detail, for the outcome ARR, CLA tablets were predicted to be less efficacious than OCR (relative risk 1.06, CI 95\% 0.78-1.45) [36].

CLA is considered an immune reconstitution therapy (IRT) [37, 38]. Characteristics of IRTs include transient reductions of B and T lymphocyte counts and/or select lymphocyte subtypes, followed by a recovery period in which the B and $\mathrm{T}$ populations gradually recover, and immune function is restored. The reconstituted lymphocyte population usually begins within weeks after each treatment course in the first and second years and stabilized on return to baseline [39-42]. Such mechanism of action could explain why the first relapse in CLA group happened between the first and second trimesters from the therapeutic switch. Such timing could coincide to partial reconstitution of different $\mathrm{T}$ cells subtypes as pooled data from clinical trials showed [38, 42-45].

A previous short report by Mohon et al. [20] analysed 17 patients switching from NTZ to CLA with a median follow-up of 9.7 months (range 1.5-15 months) [20]. No patients presented a clinical relapse during the observation period, and only 2 patients showed new T2 lesions on brain MRI. [20]. 
However, the absence of comparisons or inferential statistical models represent limits of this study.

Our observational study firstly compared 3 high-efficacy DMTs employing a generalized model IPTW PS-adjusted for baseline characteristics to mitigate unbalance among groups, and this latter certainly constitutes an element of strength of the study.

Although IPTW has not been deeply evaluated in the context of small sample sizes, simulation studies revealed that, even in case of small study samples or low prevalence of treatment, both neighbour matching and IPTW PS can yield unbiased estimations of treatment effect [46, 47].

However, our study has some limits.

As this is a retrospective study, not all participants have the same follow-up, and the characteristics of sample size warrant cautious interpretation of the data. Moreover, we did not report lymphocytic count from investigated patients, and it could have added further data about the type and timing of lymphocytic subset repopulation.

In conclusion, prospective/longitudinal studies are needed to better clarify if switching to OCR is the choice with the best risk/benefit ratio as exit strategy after NTZ interruption because of unacceptable high risk of PML.

Supplementary Information The online version contains supplementary material available at https://doi.org/10.1007/s13311-021-01037-2.

Required Author Forms Disclosure forms provided by the authors are available with the online version of this article.

Funding Open access funding provided by Università degli Studi di Catania within the CRUI-CARE Agreement.

\section{Declarations}

Protocol Approval Standard, Registrations, and Patient Consents The study protocol was approved by the local ethics committee (Comitato Etico Catania 1 no. 140/2020/PO) of the coordinating centre (Policlinico Vittorio Emanuele, Catania, Italy), and patients provided written informed consent. The study was conducted in accordance with the ethical principles of the Declaration of Helsinki and with the appropriate national regulations.

Open Access This article is licensed under a Creative Commons Attribution 4.0 International License, which permits use, sharing, adaptation, distribution and reproduction in any medium or format, as long as you give appropriate credit to the original author(s) and the source, provide a link to the Creative Commons licence, and indicate if changes were made. The images or other third party material in this article are included in the article's Creative Commons licence, unless indicated otherwise in a credit line to the material. If material is not included in the article's Creative Commons licence and your intended use is not permitted by statutory regulation or exceeds the permitted use, you will need to obtain permission directly from the copyright holder. To view a copy of this licence, visit http://creativecommons.org/licenses/by/4.0/.

\section{References}

1. Yin Y, Wang J, Wang X, et al. Prognostic value of the neutrophil to lymphocyte ratio in lung cancer: A meta-analysis. Clinics (Sao Paulo, Brazil) 2015;70:524-530.

2. Bloomgren G, Richman S, Hotermans C, et al. Risk of natalizumab-associated progressive multifocal leukoencephalopathy. $\mathrm{N}$ Engl J Med 2012;366:1870-1880.

3. D'Amico E, Zanghì A, Leone C, Tumani H, Patti F. TreatmentRelated Progressive Multifocal Leukoencephalopathy in Multiple Sclerosis: A Comprehensive Review of Current Evidence and Future Needs. Drug Safety 2016;39:1163-1174.

4. Fragoso YD, Arruda NM, Arruda WO, et al. We know how to prescribe natalizumab for multiple sclerosis, but do we know how to withdraw it? Expert review of neurotherapeutics 2014;14:127-130.

5. Ryerson LZ, Foley J, Chang I, et al. Risk of natalizumab-associated PML in patients with MS is reduced with extended interval dosing. Neurology 2019;93:e1452-e1462.

6. Sellner J, Rommer PS. A review of the evidence for a natalizumab exit strategy for patients with multiple sclerosis. Autoimmun Rev 2019;18:255-261.

7. Zhao W, Beers DR, Hooten KG, et al. Characterization of Gene Expression Phenotype in Amyotrophic Lateral Sclerosis Monocytes. JAMA neurology 2017;74:677-685.

8. Plavina T, Subramanyam M, Bloomgren G, et al. Anti-JC virus antibody levels in serum or plasma further define risk of natalizumab-associated progressive multifocal leukoencephalopathy. Annals of neurology 2014;76:802-812.

9. Alping P, Frisell T, Novakova L, et al. Rituximab versus fingolimod after natalizumab in multiple sclerosis patients. Annals of neurology 2016;79:950-958.

10. Prosperini L, Kinkel RP, Miravalle AA, Iaffaldano P, Fantaccini S. Post-natalizumab disease reactivation in multiple sclerosis: systematic review and meta-analysis. Ther Adv Neurol Disord 2019;12:1756286419837809.

11. Zhovtis Ryerson L, Frohman TC, Foley J, et al. Extended interval dosing of natalizumab in multiple sclerosis. J Neurol Neurosurg Psychiatry 2016;87:885-889.

12. Calabrese M, Pitteri M, Farina G, et al. Dimethyl fumarate: a possible exit strategy from natalizumab treatment in patients with multiple sclerosis at risk for severe adverse events. J Neurol Neurosurg Psychiatry 2017;88:1073.

13. Jokubaitis VG, Li V, Kalincik T, et al. Fingolimod after natalizumab and the risk of short-term relapse. Neurology 2014;82:1204-1211.

14. Rasenack M, Derfuss T. Disease activity return after natalizumab cessation in multiple sclerosis. Expert Rev Neurotherapeutics 2016;16:587-594.

15. D’Amico E, Patti F, Zanghì A, Zappia M. A personalized approach in progressive multiple sclerosis: the current status of disease modifying therapies (DMTs) and future perspectives. Int J Mol Sci 2016;17.

16. D'Amico E, Leone C, Zanghì A, Fermo SL, Patti F. Lateral and escalation therapy in relapsing-remitting multiple sclerosis: a comparative study. J Neurol 2016;263:1802-1809.

17. D'Amico E, Leone C, Caserta C, Patti F. Oral drugs in multiple sclerosis therapy: an overview and a critical appraisal. Expert Rev Neurotherapeutics 2015;15:803-824.

18. D'Amico E, Zanghì A, Gastaldi M, Patti F, Zappia M, Franciotta D. Placing CD20-targeted B cell depletion in multiple sclerosis therapeutic scenario: Present and future perspectives. Autoimmun Rev 2019;18:665-672.

19. Mancinelli CR, Scarpazza C, Santuccio G, De Rossi N, Capra R. Dealing with highly active multiple sclerosis after natalizumab- 
associated PML: could rituximab be of help? Neurological sciences : official journal of the Italian Neurological Society and of the Italian Society of Clinical Neurophysiology 2018;39:965-966.

20. Möhn N, Skripuletz T, Sühs KW, Menck S, Voß E, Stangel M. Therapy with cladribine is efficient and safe in patients previously treated with natalizumab. Ther Adv Neurol Disord 2019;12:1756286419887596.

21. Thompson AJ, Banwell BL, Barkhof F, et al. Diagnosis of multiple sclerosis: 2017 revisions of the McDonald criteria. The Lancet Neurology 2018;17:162-173.

22. Broce I, Karch CM, Wen N, et al. Immune-related genetic enrichment in frontotemporal dementia: An analysis of genome-wide association studies. PLoS medicine 2018;15:e1002487.

23. https://www.ema.europa.eu/en/documents/product-information/ mabthera-epar-product-information_it.pdf.

24. Ellwardt E, Ellwardt L, Bittner S, Zipp F. Monitoring B-cell repopulation after depletion therapy in neurologic patients. Neurology - Neuroimmunology Neuroinflammation 2018;5:e463.

25. Moreau C, Devos D, Brunaud-Danel V, et al. Elevated IL-6 and TNF-alpha levels in patients with ALS: inflammation or hypoxia? Neurology 2005;65:1958-1960.

26. Giovannoni G, Turner B, Gnanapavan S, Offiah C, Schmierer $\mathrm{K}$, Marta M. Is it time to target no evident disease activity (NEDA) in multiple sclerosis? Multiple sclerosis and related disorders 2015;4:329-333.

27. Clerico M, De Mercanti SF, Signori A, et al. Extending the Interval of Natalizumab Dosing: Is Efficacy Preserved? Neurotherapeutics : J Amer Soc Exper NeuroTherapeutics 2020;17:200-207.

28. Auger J, Sermondade N, Eustache F. Semen quality of 4480 young cancer and systemic disease patients: baseline data and clinical considerations. Basic Clin Androl 2016;26:3.

29. Scarpazza C, Signori A, Prosperini L, et al. Early diagnosis of progressive multifocal leucoencephalopathy: longitudinal lesion evolution. J Neurol Neurosurg Psychiatry 2019;90:261.

30. Hodel J, Outteryck O, Dubron C, et al. Asymptomatic Progressive Multifocal Leukoencephalopathy Associated with Natalizumab: Diagnostic Precision with MR Imaging. Radiology 2016;278:863-872.

31. Kivisäkk P, Healy BC, Viglietta V, et al Natalizumab treatment is associated with peripheral sequestration of proinflammatory $\mathrm{T}$ cells. Neurology 2009;72:1922-1930.

32. D'Amico E, Zanghì A, Chisari CG, et al. Effectiveness and safety of Rituximab in demyelinating diseases spectrum: An Italian experience. Multi Scler Relat Dis 2019;27:324-326.

33. Malucchi S, Capobianco M, di Sapio A, Re ML, Cavalla P, Bertolotto A. Rituximab suppresses disease activity after natalizumab withdrawal: an exploratory study. Multi Scler Demyelinating Dis 2016;1:11.

34. Mancinelli CR, Scarpazza C, Cordioli C, et al. Switching to ocrelizumab in RRMS patients at risk of PML previously treated with extended interval dosing of natalizumab. Multi Scler J 2020:1352458520946017.
35. Ng HS, Rosenbult CL, Tremlett H. Safety profile of ocrelizumab for the treatment of multiple sclerosis: a systematic review. Expert Opin Drug Saf 2020;19:1069-1094.

36. Berardi A, Siddiqui MK, Treharne C, Harty G, Wong SL. Estimating the comparative efficacy of cladribine tablets versus alternative disease modifying treatments in active relapsing-remitting multiple sclerosis: adjusting for patient characteristics using meta-regression and matching-adjusted indirect treatment comparison approaches. Curr Med Res Opin 2019;35:1371-1378.

37. Boyko AN, Boyko OV. Cladribine tablets' potential role as a key example of selective immune reconstitution therapy in multiple sclerosis. Degener Neurol Neuromuscul Dis 2018;8:35-44.

38. Comi G, Cook S, Giovannoni G, et al. Effect of cladribine tablets on lymphocyte reduction and repopulation dynamics in patients with relapsing multiple sclerosis. Multi Scler Rel Dis 2019;29:168-174.

39. Ceronie B, Jacobs BM, Baker D, et al. Cladribine treatment of multiple sclerosis is associated with depletion of memory B cells. J Neurol 2018;265:1199-1209.

40. Wiendl H. Cladribine - an old newcomer for pulsed immune reconstitution in MS. Nat Rev Neurol 2017;13:573-574.

41. Comi G, Cook S, Rammohan K, et al. Long-term effects of cladribine tablets on MRI activity outcomes in patients with relapsing-remitting multiple sclerosis: the CLARITY Extension study. Ther Adv Neurol Disord 2018;11:1756285617753365.

42. Giovannoni G, Soelberg Sorensen P, Cook S, et al. Safety and efficacy of cladribine tablets in patients with relapsing-remitting multiple sclerosis: Results from the randomized extension trial of the CLARITY study. Multiple sclerosis (Houndmills, Basingstoke, England) 2018;24:1594-1604.

43. Bar-Or A, Fawaz L, Fan B, et al. Abnormal B-cell cytokine responses a trigger of T-cell-mediated disease in MS? Annals of neurology 2010;67:452-461.

44. Palanichamy A, Jahn S, Nickles D, et al. Rituximab efficiently depletes increased CD20-expressing T cells in multiple sclerosis patients. J Immunol (Baltimore, Md : 1950) 2014;193:580-586.

45. Schuh E, Berer K, Mulazzani M, et al. Features of human $\mathrm{CD} 3+\mathrm{CD} 20+\mathrm{T}$ cells. J Immunol (Baltimore, Md : 1950) 2016;197:1111-1117.

46. Rubin DB, Thomas N. Matching using estimated propensity scores: relating theory to practice. Biometrics 1996;52:249-264.

47. Weitzen S, Lapane KL, Toledano AY, Hume AL, Mor V. Weaknesses of goodness-of-fit tests for evaluating propensity score models: the case of the omitted confounder. Pharmacoepidemiology Drug Saf 2005;14:227-238.

Publisher's Note Springer Nature remains neutral with regard to jurisdictional claims in published maps and institutional affiliations. 


\section{Authors and Affiliations}

\section{Aurora Zanghì ${ }^{1}$ (1) - Antonio Gallo ${ }^{2} \cdot$ Carlo Avolio $^{3} \cdot$ Rocco Capuano $^{2} \cdot$ Matteo Lucchini $^{4} \cdot$ Maria Petracca $^{7}$. Simona Bonavita ${ }^{6} \cdot$ Roberta Lanzillo $^{7}$. Diana Ferraro ${ }^{8}$. Erica Curti ${ }^{9} \cdot$ Maria Buccafusca $^{10}$. Graziella Callari ${ }^{11}$. Stefania Barone ${ }^{12}$. Giuseppe Pontillo ${ }^{13,14}$. Gianmarco Abbadessa ${ }^{15}$. Valeria Di Francescantonio ${ }^{3}$. Elisabetta Signoriello ${ }^{16}$. Giacomo Lus ${ }^{16} \cdot$ Patrizia Sola $^{8}$. Franco Granella ${ }^{17,9} \cdot$ Paola Valentino $^{12}$. Massimiliano Mirabella ${ }^{4,5}$. Francesco Patti ${ }^{1} \cdot$ Emanuele D'Amico $^{1}$ (1)}

1 Department "G.F. Ingrassia", MS Center, Organization University of Catania, Catania, Italy

2 MS Center I Division of Neurology, University Della Campania “L. Vanvitelli”, Naples, Italy

3 Department of Medical and Surgical Sciences Head of Multiple Sclerosis Center Dept. of Neurosciences, University of Foggia, Foggia, Italy

4 Fondazione Policlinico Universitario “A. Gemelli” IRCCS, Istituto di Neurologia, Università Cattolica del Sacro Cuore, Rome, Italy

5 Università Cattolica del Sacro Cuore, Rome, Italy

6 Dipartimento Di Scienze Mediche E Chirurgiche Avanzate, Università Della Campania Luigi Vanvitelli, Piazza Miraglia, 2, 80138 Naples, Italy

7 Department of Neuroscience, Reproductive Sciences and Odontostomatology, University of Naples "Federico II", Naples, Italy

8 University of Modena and Reggio Emilia, Modena, Italy

9 Multiple Sclerosis Centre, Department of General Medicine, Parma University Hospital, Parma, Italy
10 Azienda Ospedaliera Universitaria “G. Martino”, Messina, Italy

11 Institute Foundation “G. Giglio”, Cefalù, Italy

12 Azienda Ospedaliera Universitaria "Mater Domini”, Catanzaro, Italy

13 Department of Advanced Biomedical Sciences, University "Federico II", Naples, Italy

14 Department of Electrical Engineering and Information Technology, , University “Federico II", Naples, Italy

15 Dipartimento Di Scienze Mediche E Chirurgiche Avanzate, Università Della Campania Luigi Vanvitelli, Piazza Miraglia, 2, 80138 Napoli, Italy

16 Department of Clinical and Experimental Medicine, Multiple Sclerosis Center, II Division of Neurology, Second University of Naples, Naples, Italy

17 Unit of Neurosciences, Department of Medicine and Surgery, University of Parma, Parma, Italy 